\title{
Potential of the cell-free blood-based biomarker uroplakin 2 RNA to detect recurrence after surgical resection of lung adenocarcinoma
}

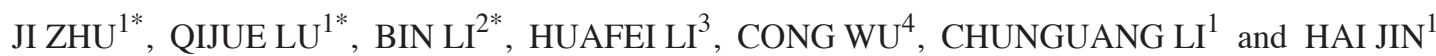 \\ ${ }^{1}$ Department of Thoracic Surgery, First Affiliated Hospital of The Second Military Medical University, Shanghai 200433; \\ ${ }^{2}$ Department of Thoracic Surgery, Section of Esophageal Surgery, Shanghai Chest Hospital, Shanghai Jiao Tong University, \\ Shanghai 200030; ${ }^{3}$ School of Life Sciences, Shanghai University, Shanghai 200444; ${ }^{4}$ Department of Laboratory Diagnosis, \\ First Affiliated Hospital of The Second Military Medical University, Shanghai 200433, P.R. China
}

Received September 4, 2019; Accepted October 26, 2020

DOI: $10.3892 / \mathrm{ol} .2021 .12781$

\begin{abstract}
Lung adenocarcinoma (LUAD) is the most common subtype of lung cancer, and $30 \%$ of patients with LUAD develop cancer recurrence after surgery. The present study aimed to identify and validate biomarkers that may be used to monitor recurrence following LUAD surgery. Data from patients with LUAD were downloaded from The Cancer Genome Atlas database and postoperative recurrence samples were selected. Subsequently, weighted gene co-expression network analysis (WGCNA) was subsequently performed to identify key co-expression gene modules. Additionally, enrichment analysis of the key gene modules was performed using the Database for Annotation, Visualization and Integrated Discovery. Furthermore, survival analysis was performed on the most notable biomarker, uroplakin 2 (UPK2), which was downloaded from the Oncomine database, and its effect on prognosis was assessed. WGCNA identified 39 gene modules, of which one was most associated with recurrence. Among them, UPK2, kelch domain containing 3, galanin receptor 2 and tyrosinase-related protein 1 served a central role in the
\end{abstract}

Correspondence to: Dr Chunguang Li or Dr Hai Jin, Department of Thoracic Surgery, First Affiliated Hospital of The Second Military Medical University, 168 Changhai Road, Yangpu, Shanghai 200433, P.R. China

E-mail: dr_lichunguang@163.com

E-mail:projinhai@163.com

"Contributed equally

Abbreviations: LUAD, lung adenocarcinoma; TCGA, The Cancer Genome Atlas; WGCNA, weighted gene co-expression network analysis; DAVID, Database for Annotation, Visualization and Integrated Discovery; ULPK2, uroplakin 2; KLHDC3, kelch domain containing 3; GALR2, galanin receptor 2; TYRP1, tyrosinase-related protein 1; GO, Gene Ontology

Key words: LUAD, TCGA database, WGCNA, plasma, UPK2 co-expression network and were significantly associated with the survival of patients. A total of 132 blood samples were collected from patients with LUAD with free UPK2 in the plasma. The expression levels of UPK2 relative to GADPH were 0.1623 and 0.2763 in non-relapsed and relapsed patients, respectively. Receiver operating characteristic curve analysis was used to detect free UPK2 mRNA in the blood in order to monitor postoperative recurrence, resulting in an area under the curve of 0.767 and a $95 \%$ CI of $0.675-0.858$. Patients with high free UPK2 mRNA expression had unfavorable survival outcomes compared with those with low UPK2 expression. Therefore, free UPK 2 mRNA expression in the plasma may have the potential to act as an indicator of postoperative recurrence in patients with early stage LUAD.

\section{Introduction}

Non-small cell lung cancer (NSCLC) accounts for $\sim 85 \%$ of all diagnosed cases of lung cancer (1). Lung adenocarcinoma (LUAD) is the most common subtype of NSCLC, and its morbidity and mortality rates have increased to 0.057 and 85\%, respectively, in China in 2017 (2,3). Advancements in science and technology have allowed the development of novel therapeutic strategies for patients with lung cancer. For example, Han et al (4) discovered that pemetrexed plus carboplatin combined with gefitinib prolongs the survival time of patients with LUAD who harbor sensitive EGFR mutations. Furthermore, Zhuang et al (5) demonstrated that combination treatment of nadroparin with radiotherapy induces stronger synergistic antitumor effects in LUAD A549 cells. However, recent studies suggest that current treatment strategies can be further improved (6-8).

Despite advancements in surgery, molecular subtyping and targeted therapy, the prognosis of patients with LUAD remains relatively poor (9). Patients with LUAD often relapse and develop metastases following surgery, chemotherapy and radiotherapy (10). Due to its malignant characteristics, the 5-year survival rate of patients with early stage LUAD is $50-70 \%$ (11). Patients with advanced LUAD are often resistant to conventional chemotherapies or targeted therapeutic 
drugs (11). Furthermore, high-potency anticancer drugs remain ineffective for long-term use, and cancer cells become resistant to anticancer drugs as the mutation rate rapidly increases (12). Despite consistent improvements to the surgical methods for treating cancer, and regular updates to chemotherapy drugs, the complete removal of residual cancer cells remains difficult, and thus, the risk of recurrence remains relatively high (13). The recurrence of cancer proves problematic to subsequent treatment strategies, and the rate of deterioration increases (13). Furthermore, the molecular mechanisms underlying recurrence have not been fully elucidated. A previous study reported that $85 / 289$ patients with stage I and II LUAD developed distant recurrence within 5 years (14), suggesting that cancer recurrence holds important clinical value.

With the development of precision medicine, several biomarkers have been demonstrated to be positively associated with biological events. For example, Krishnamurthy et al (15) reported that cyclin-dependent kinase inhibitor $2 \mathrm{~A}$ (CDKN2A) expression is a biomarker of aging, with high CDKN2A expression being associated with advanced aging in rodent tissues. In addition, $\beta$-amyloid 1-42 in cerebrospinal fluid (CSF) has been verified as a biomarker for Alzheimer's disease in the autopsy cohort of CSF samples, with a high sensitivity for detection of $96.4 \%$ (16). These biomarkers are not just limited to proteins, since mRNAs can also act as biomarkers. Ji et al (17) demonstrated that microRNA-208 is a useful indicator of myocardial injury. Notable progress has been made in the discovery and verification of tumor biomarkers, particularly in the discovery of molecular markers associated with the clinical effects of tumor therapy. In 2011, human epididymis protein 4 was approved by the Food and Drug Administration to monitor the recurrence or disease progression of epithelial ovarian cancer in conjunction with CA125 (18). Huang et al (19) reported that abnormalities of amplified in breast cancer 1 (AIB1) are significantly associated with prognostic significance in urothelial carcinoma, and high AIB1 expression is associated with increased hazard ratios for the 5-year cause-specific survival rate (80.6 vs. 55.8\% for high and low AIB1 expression, respectively; $\mathrm{P}=0.008)$ and 5-year overall survival rate (78.1 vs. $54.8 \%$ for high and low AIB1 expression, respectively; $\mathrm{P}=0.006$ ). The homeobox B13/IL17 receptor B biomarker predicts the recurrence risk in estrogen receptor-positive and lymph node-negative patients with breast cancer (20). Previous studies have identified several biomarkers that are currently applied in clinical settings $(21,22)$.

The present study performed bioinformatics analysis to assess a robust sequence of data, and several biomarkers were verified using clinical samples of LUAD. Further evaluations were performed to verify whether cell-free UPK 2 mRNA may be used as a potential biomarker for postoperative recurrence in patients with early stage LUAD.

\section{Materials and methods}

Data analysis. The datasets used in the present study were downloaded from The Cancer Genome Atlas (TCGA) database (https://www.cancer.gov/about-nci/organization/ccg/ research/structural-genomics/tcga; Project ID, TCGA-LUAD). The transcriptome data of 24 relapsed patients, including 14 men and 10 women, was screened. Additionally, the transcriptome data of paracancerous tissues from 53 patients (33 men and 20 women) was included. The soft connectivity function in the weighted gene co-expression network analysis (WGCNA; v1.69; https://cran.r-project.org/web/packages/WGCNA/index. html) package (23) was used to assess the effects of different power values on the co-expression network and co-expression modules in the scale independence and average connectivity by Pearson's correlation analysis. The 'randomly selected gene' parameter was set to 5,000, and all other parameters were set to the default values. The expression values were summarized using the collapse rows function in the WGCNA package, and cluster analysis was subsequently performed using flashClust v1.01-2 (https://cran.r-project.org/web/packages/flashClust/index. html) (24). The interaction/association of each module was visualized using heat maps. In addition, Gene Ontology (GO) analysis of the gene modules of interest was performed using the Database for Annotation, Visualization and Integrated Discovery (DAVID; https://david.ncifcrf.gov/summary.jsp). The differential expression of genes was defined as $\log$ (fold-change) $\mid<0.61$; false discovery rate $<0.05$. Cytoscape v3.11 was used to construct co-expression network (https://cytoscape.org/download.html). Kaplan-Meier survival analysis of the corresponding genes was performed using the Oncomine database (https://www.oncomine. org/resource/login.html) and the P-values were calculated using the log-rank test.

Patient recruitment. Blood samples were collected from 132 patients with LUAD who underwent excision surgery at the First Affiliated Hospital of the Second Military Medical University (Shanghai, China) between February 2006 and March 2010. All patients received systematic treatment following surgery, including optimal local treatments, such as radiochemotherapy and targeted therapy. Disease recurrence was based on chest X-ray or CT imaging. The present study was performed in accordance with the Declaration of Helsinki and Good Clinical Practice, and was approved by the Ethics Committee of the First Affiliated Hospital of the Second Military Medical University. All patients provided oral informed consent for the use of their blood for scientific purposes.

Sample collection and processing. Blood samples $(15 \mathrm{ml})$ from 105 patients with early stage LUAD of the 132 patients (pathological stage I, II and IIIA) were initially collected 90 days after surgery. The second, third and fourth samples were collected 180 days, 1 year and 2 years after surgery, respectively. Blood samples were no longer collected if disease recurrence was detected during repeated examinations. The samples were collected in 5-ml heparin anticoagulation tubes and immediately used to extract free mRNA according to the manufacturer's protocol (Qiagen, Inc.; cat. no. 72022).

Free mRNA was reverse transcribed using the reverse transcription (RT) kit according to the manufacturer's protocol (Takara Bio, Inc.; cat. no. 639522). A quarter of the RT product was mixed with a pre-formulated 2X SYBR Green PCR mix (Roche Diagnostics; cat. no. 06924204001) containing uroplakin 2 (UPK2) RT-quantitative (q)PCR primers (UPK2 forward, 5'-CACTGAGTCCAGCAGAGAGATC-3' and reverse, 5'-ACAGAGAGCAGCACCGTGATGA-3'; GAPDH 
forward, 5'-GTCTCCTCTGACTTCAACAGCG-3' and reverse, 5'-ACCACCCTGTTGCTGTAGCCAA-3') and was subsequently supplemented with distilled water to reach a final volume of $20 \mu \mathrm{l}$. Amplification was performed to obtain the dissolution curve, using the following thermocycling conditions: $94^{\circ} \mathrm{C}$ for $5 \mathrm{~min}$, followed by 40 cycles at $94^{\circ} \mathrm{C}$ for $2 \mathrm{~min}$, $60^{\circ} \mathrm{C}$ for $1 \mathrm{~min}$ and $72^{\circ} \mathrm{C}$ for $2 \mathrm{~min}$, and a final extension step at $72^{\circ} \mathrm{C}$ for $2 \mathrm{~min}$. Relative UPK 2 expression was calculated using the $2^{-\Delta \Delta \mathrm{Cq}}$ method (25) and normalized to the internal reference gene GAPDH.

Statistical analysis. The serum expression levels of UPK2 were detected in triplicate. GraphPad Prism 8.0 (GraphPad Software Inc.) was used for statistical analysis. The data are presented as the mean $\pm \mathrm{SD}$. The difference between two groups was analyzed by unpaired Student's t-test. The difference between paired samples (before and after relapse) was analyzed by paired Student's t-test. $\mathrm{P}<0.05$ was considered to indicate a statistically significant difference. The receiver operating characteristic (ROC) curve of LUAD recurrence was assessed using the pROC package v1.16.2 (https://cran.r-project.org/web/packages/pROC/index.html) (26) within $\mathrm{R}$ software, and the area under the curve (AUC) was calculated. The survival curve was plotted using the ggsurvplot package v0.4.8 (https://cran.r-project. org/web/packages/survminer/readme/README.html) within $\mathrm{R}$ language and the log-rank test was used to obtain the P-value.

\section{Results}

Specific co-expression modules associated with LUAD recurrence. In order to establish a co-expressed gene network associated with postoperative recurrence of LUAD, WGCNA was used to assess the gene expression profile data from patients with recurrent LUAD within the TCGA database. The transcriptome data of 24 relapsed patients, including 14 men and 10 women, were screened. The association between genes was calculated using WGCNA, and the gene expression data of patients with recurrent LUAD were classified into 39 gene modules using unsupervised average linkage hierarchical clustering and were labelled in a heat map with different colors (Fig. 1A). Gene modules of different colors contained mutually exclusive co-expressed genes. Genes that could not be classified into a specific module were incorporated into gray modules. WGCNA can determine the association between gene modules and a series of phenotypes (23); therefore, this method was used to assess the association between the specific gene modules of patients with postoperative recurrent early stage LUAD and a series of phenotypes, including age, sex, survival, recurrence, recurrence type and pathological stage (27). Without any phenotypic or genetic preferences for module partitioning, the results demonstrated that the purple module was significantly associated with survival and recurrence, with Pearson's correlation coefficients $>0.7$ (Fig. 1B). Overall, the present results suggested that these genes and their co-expression patterns may be associated with the recurrence of LUAD.

Biological insights from the purple module. WGCNA classifies co-expressed genes of patient samples into specific modules associated with a series of traits regulated by the same mechanism (23). The purple module was identified as the most relevant to postoperative recurrence. In order to verify the association between the co-expressed genes within the purple module and LUAD, a heat map containing the gene expression levels of 24 recurrent tumor tissues and 53 paracancerous tissues from TCGA was constructed. The results demonstrated a marked difference in the expression pattern of the purple module between paracancerous and recurrent tumor tissues (Fig. 2A). However, the expression pattern of the purple module genes in patients with recurrent tumor tissues was not as consistent compared with paracancerous tissues; the expression pattern in tumor tissues exhibited three expression patterns of light red, light blue and deep red, suggesting different mechanisms of relapse (Fig. 2A). Of the 117 genes in the purple module, 68 genes exhibited significant differences (data not shown) between recurrent tumor tissues and paracancerous tissues $[\log ($ fold-change $) \mid<0.61$; false discovery rate $<0.05]$. These 68 genes were used to construct an expression heat map of the two types of tissues (Fig. 2C), which demonstrated that the expression patterns of the 68 genes were more uniform than that of the heat map constructed using 117 genes. The biological function of the 117 genes in the purple module was further analyzed via GO analysis using DAVID, with the most significant GO term being 'cytosol' ( $\mathrm{P}=0.0356$; Fig. 2B).

Key genes associated with LUAD recurrence in the purple module. Gene significance is closely associated with gene connectivity, which means that nodes with higher connectivity in the co-expression network serve an important role in the process of performing biological functions (28). Therefore, a co-expression network of genes for LUAD recurrence was constructed, identifying 47 edges and 17 nodes (power $=8$; Fig. 3A). The results demonstrated that there were four genes [UPK2, kelch domain containing 3 (KLHDC3), galanin receptor 2 (GALR2) and tyrosinase-related protein 1 (TYRP1)] in the co-expression network with more nodes linked appearing in the purple module, which were significantly associated with survival and recurrence (Table I and Fig. 3B-E). Among these genes, high expression levels of UPK2, KLHDC 3 and GALR2, and low expression levels of TYRP1 were associated with a poor prognosis (Table I and Fig. 3B-E). The function of these four genes in LUAD was further analyzed using clinical data downloaded from the Oncomine database. The results demonstrated that patients with low expression levels of UPK2, KLHDC3 and GALR2 had significantly improved survival outcomes than those with high expression levels of UPK2, KLHDC 3 and GALR2 $\left(\mathrm{P}=4.9 \times 10^{-5}, \mathrm{P}=1.7 \times 10^{-5}\right.$ and $\mathrm{P}=0.0093$, respectively; Fig. 3B-D). Conversely, patients with high TYRP1 expression had a significantly improved prognosis than those with low TYRP1 expression ( $\mathrm{P}=2 \times 10^{-7}$; Fig. 3E).

Clinical characteristics of patients with early stage LUAD who received UPK2 free mRNA plasma testing. Previous studies have reported that free mRNA in plasma has the potential to act as a tumor marker $(29,30)$. Table II presents the demographic information and clinical characteristics of the 105 patients with early stage LUAD who met the study criteria out of 132 patients. Of these LUAD patients, 58 were men $(55 \%)$ and 47 were women $(45 \%)$, with a median age of 
A
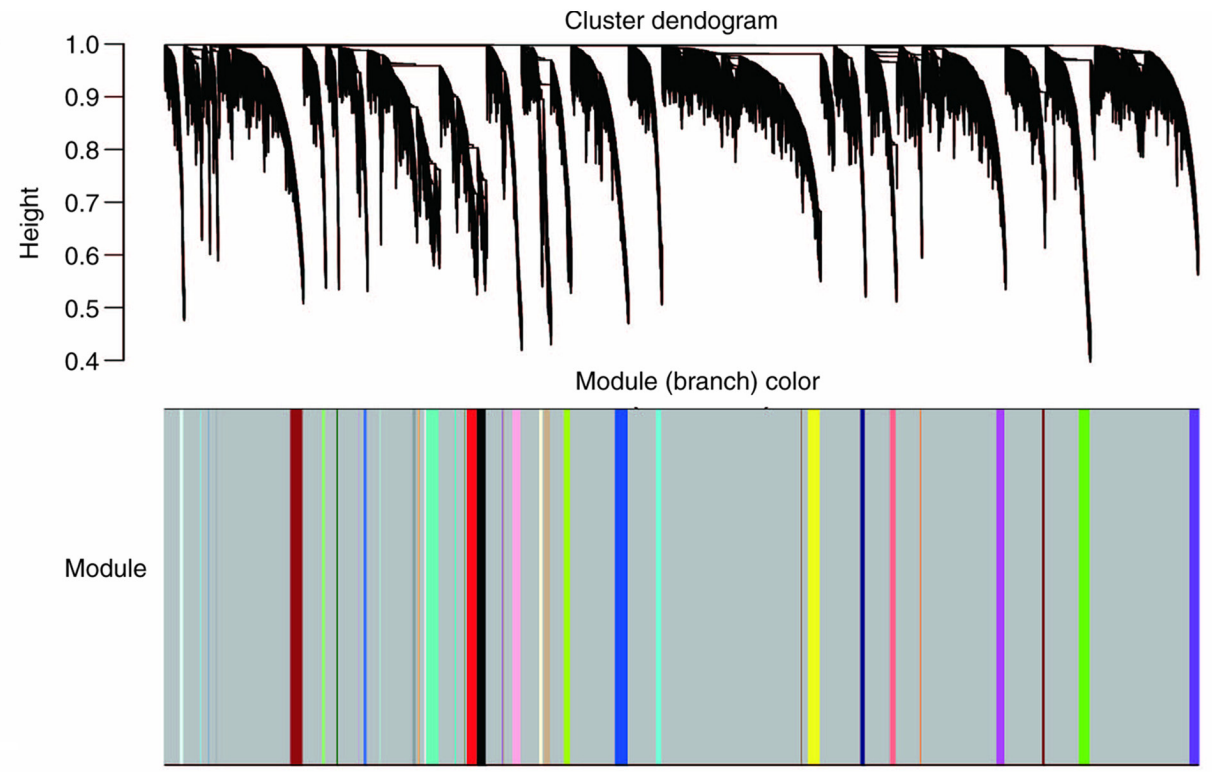

B

\begin{tabular}{|c|c|c|c|c|c|c|c|}
\hline \multicolumn{7}{|c|}{ Module-trait relationships } & \\
\hline \multirow{2}{*}{$\begin{array}{l}\text { MEdarkred } \\
\text { MEsalmon }\end{array}$} & $0.066(0.8)$ & $-0.3(0.2)$ & $-0.13(0.5)$ & $-0.13(0.5)$ & $0.014(0.9)$ & $-0.22(0.3)$ & \\
\hline & $0.23(0.3)$ & $-0.27(0.2)$ & $0.19(0.4)$ & $-0.00074(1)$ & $0.29(0.2)$ & $-0.26(0.2)$ & \\
\hline MEmagenta & $-0.23(0.3)$ & $0.15(0.5)$ & $-0.18(0.4)$ & $-0.081(0.7)$ & $-0.016(0.9)$ & $-0.24(0.3)$ & \multirow{10}{*}{-0.5} \\
\hline MEdarkorange & $0.41(0.04)$ & $-0.26(0.2)$ & $0.029(0.9)$ & $-0.031(0.9)$ & $-0.25(0.2)$ & $-0.22(0.3)$ & \\
\hline MEgreen & $-0.078(0.7)$ & $-0.27(0.2)$ & $-0.029(0.9)$ & $-0.12(0.6)$ & $-0.24(0.3)$ & $0.028(0.9)$ & \\
\hline MEmidnightblue & $-0.2(0.3)$ & $0.2(0.4)$ & $0.092(0.7)$ & $-0.033(0.9)$ & $-0.22(0.3)$ & $-0.21(0.3)$ & \\
\hline MEsaddlebrown & $-0.11(0.6)$ & $0.25(0.2)$ & $0.35(0.1)$ & $0.35(0.09)$ & $0.14(0.5)$ & $-0.32(0.1)$ & \\
\hline \multirow{2}{*}{$\begin{array}{l}\text { MEyellow } \\
\text { MEpurple }\end{array}$} & $0.031(0.9)$ & $0.2(0.3)$ & $0.17(0.4)$ & $0.19(0.4)$ & $0.3(0.2)$ & $-0.21(0.3)$ & \\
\hline & $-0.15(0.5)$ & $0.18(0.4)$ & $0.72\left(8 \times 10^{-5}\right)$ & $0.91\left(8 \times 10^{10}\right)$ & $-0.23(0.3)$ & $-0.24(0.3)$ & \\
\hline \multirow{2}{*}{$\begin{array}{c}\text { MEyellowgreen } \\
\text { MEbrown }\end{array}$} & $0.32(0.1)$ & $0.26(0.2)$ & $0.11(0.6)$ & $0.059(0.8)$ & $-0.14(0.5)$ & $-0.012(1)$ & \\
\hline & $0.26(0.2)$ & $0.18(0.4)$ & $0.25(0.2)$ & $0.13(0.5)$ & $-0.25(0.2)$ & $-0.19(0.4)$ & \\
\hline MEplum1 & $0.14(0.5)$ & $0.26(0.2)$ & $0.58(0.003)$ & $0.54(0.007)$ & $-0.32(0.1)$ & $-0.27(0.2)$ & \\
\hline MEsteelblue & $0.052(0.8)$ & $-0.26(0.2)$ & $-0.16(0.5)$ & $-0.21(0.3)$ & $0.041(0.8)$ & $0.3(0.2)$ & \multirow{9}{*}{-0} \\
\hline MEgreenyellow & $-0.42(0.04)$ & $-0.21(0.3)$ & $-0.2(0.4)$ & $-0.16(0.5)$ & $-0.26(0.2)$ & $0.027(0.9)$ & \\
\hline \multirow{2}{*}{$\begin{array}{r}\text { MElightyellow } \\
\text { MEtan }\end{array}$} & $0.18(0.4)$ & $0.11(0.6)$ & $-0.15(0.5)$ & $-0.13(0.6)$ & $-0.067(0.8)$ & $0.25(0.2)$ & \\
\hline & $-0.11(0.6)$ & $-0.14(0.5)$ & $-0.24(0.3)$ & $-0.22(0.3)$ & $-0.29(0.2)$ & $0.16(0.4)$ & \\
\hline \multirow{2}{*}{$\begin{array}{r}\text { MEblue } \\
\text { MEcyan }\end{array}$} & $-0.44(0.03)$ & $0.18(0.4)$ & $-0.29(0.2)$ & $-0.19(0.4)$ & $0.32(0.1)$ & $0.52(0.009)$ & \\
\hline & $-0.12(0.6)$ & $0.2(0.4)$ & $-0.22(0.3)$ & $-0.14(0.5)$ & $0.1(0.6)$ & $0.11(0.6)$ & \\
\hline \multirow{2}{*}{$\begin{array}{l}\text { MEdarkgrey } \\
\text { MEdarkgreen }\end{array}$} & $0.13(0.5)$ & $-0.22(0.3)$ & $-0.2(0.3)$ & $-0.13(0.5)$ & $0.048(0.8)$ & $0.075(0.7)$ & \\
\hline & $-0.14(0.5)$ & $0.2(0.4)$ & $0.07(0.7)$ & $-0.071(0.7)$ & $0.3(0.1)$ & $0.26(0.2)$ & \\
\hline MElightgreen & $0.022(0.9)$ & $0.19(0.4)$ & $-0.11(0.6)$ & $-0.044(0.8)$ & $0.025(0.9)$ & $0.076(0.7)$ & \\
\hline MElightcyan & $0.25(0.2)$ & $0.18(0.4)$ & $-0.022(0.9)$ & $0.043(0.8)$ & $0.023(0.9)$ & $-0.21(0.3)$ & \multirow{8}{*}{--0.5} \\
\hline MEpink & $0.2(0.3)$ & $0.2(0.4)$ & $0.15(0.5)$ & $0.08(0.7)$ & $0.3(0.2)$ & $0.26(0.2)$ & \\
\hline \multirow{2}{*}{$\begin{array}{r}\text { MEdarkmagenta } \\
\text { MEsienna3 }\end{array}$} & $0.38(0.07)$ & $0.19(0.4)$ & $0.017(0.9)$ & $-0.00081(1)$ & $0.45(0.03)$ & $0.098(0.6)$ & \\
\hline & $0.3(0.2)$ & $0.28(0.2)$ & $-0.042(0.8)$ & $-0.036(0.9)$ & $0.41(0.05)$ & $0.2(0.4)$ & \\
\hline \multirow{2}{*}{$\begin{array}{l}\text { MEpaleturquoise } \\
\text { MEdarkturquoise }\end{array}$} & $0.16(0.4)$ & $-0.24(0.3)$ & $-0.16(0.4)$ & $-0.1(0.6)$ & $-0.27(0.2)$ & $0.28(0.2)$ & \\
\hline & $-0.014(0.9)$ & $-0.086(0.7)$ & $-0.096(0.7)$ & $-0.12(0.6)$ & $0.004(1)$ & $0.23(0.3)$ & \\
\hline \multirow{2}{*}{$\begin{array}{r}\text { MEdarkolivegreen } \\
\text { MEblack }\end{array}$} & $-0.11(0.6)$ & $0.22(0.3)$ & $-0.22(0.3)$ & $-0.13(0.5)$ & $-0.12(0.6)$ & $0.021(0.9)$ & \\
\hline & $-0.053(0.8)$ & $0.044(0.8)$ & $-0.051(0.8)$ & $-0.064(0.8)$ & $-0.25(0.2)$ & $0.16(0.5)$ & \\
\hline MEred & $-0.036(0.9)$ & $0.1(0.6)$ & $-0.00073(1)$ & $-0.0032(1)$ & $-0.23(0.3)$ & $0.11(0.6)$ & \\
\hline \multirow{2}{*}{ MEorange } & $0.23(0.3)$ & $-0.21(0.3)$ & $-0.19(0.4)$ & $-0.19(0.4)$ & $0.28(0.2)$ & $0.1(0.6)$ & \\
\hline & $0.17(0.4)$ & $0.052(0.8)$ & $-0.042(0.8)$ & $-0.088(0.7)$ & $-0.052(0.8)$ & $0.13(0.5)$ & \\
\hline $\begin{array}{r}\text { MEgrey60 } \\
\text { MEskyblue3 }\end{array}$ & $0.097(0.7)$ & $-0.035(0.9)$ & $-0.2(0.3)$ & $-0.2(0.3)$ & $0.11(0.6)$ & $0.24(0.3)$ & \\
\hline \multirow{2}{*}{$\begin{array}{r}\text { MEturquoise } \\
\text { MEwhite }\end{array}$} & $0.27(0.2)$ & $-0.022(0.9)$ & $-0.17(0.4)$ & $-0.17(0.4)$ & $0.2(0.3)$ & $0.2(0.3)$ & \\
\hline & $0.35(0.09)$ & $0.0036(1)$ & $-0.11(0.6)$ & $-0.14(0.5)$ & $0.11(0.6)$ & $0.14(0.5)$ & \\
\hline \multirow{2}{*}{$\begin{array}{l}\text { MEskyblue } \\
\text { MEroyalblue }\end{array}$} & $0.036(0.9)$ & $-0.26(0.2)$ & $0.15(0.5)$ & $-0.02(0.9)$ & $-0.23(0.3)$ & $0.0067(1)$ & \\
\hline & $-0.11(0.6)$ & $0.21(0.3)$ & $-0.086(0.7)$ & $-0.036(0.9)$ & $0.051(0.8)$ & $0.037(0.9)$ & -1 \\
\hline MEviolet & $-0.16(0.5)$ & $0.034(0.9)$ & $0.013(1)$ & $-0.041(0.8)$ & $-0.22(0.3)$ & $0.049(0.8)$ & \\
\hline MEgrey & $-0.31(0.1)$ & $0.014(0.9)$ & $0.063(0.8)$ & $0.18(0.4)$ & $0.079(0.7)$ & $-0.24(0.3)$ & \\
\hline & Age & Sex & Survival & Recurren & $\begin{array}{l}\text { Recurrent } \\
\text { type }\end{array}$ & Stage & \\
\hline
\end{tabular}

Figure 1. Gene clusters and gene modules. (A) Gene dendrogram obtained by average linkage hierarchical clustering. (B) Heat map depicting the association between gene modules and phenotypes. The red/green scale bar represent the Pearson's correlation coefficients between modules and phenotypes. The Pearson's correlation weights are labeled in separate boxes and the positive numbers represent a positive correlation and negative numbers a negative correlation between the gene module and the phenotype. The greater the absolute value of the weight, and the darker the color of the square, the stronger the association. The values in parentheses represent the P-values of the correlation coefficient.

58 years (age range, 39-83 years). The pathological stage of most patients was stage I or II (83\%), whilst the remaining patients were at stage IIIa (17\%). Following surgery, 43 patients received adjuvant therapy (41\%), including 35 patients receiving radiotherapy, 4 patients receiving adjuvant chemotherapy and 4 patients receiving both radiotherapy and chemotherapy (Table II).

Diagnostic performance of UPK2. Patient blood samples were collected and assessed for free UPK2 expression. Imaging 
A

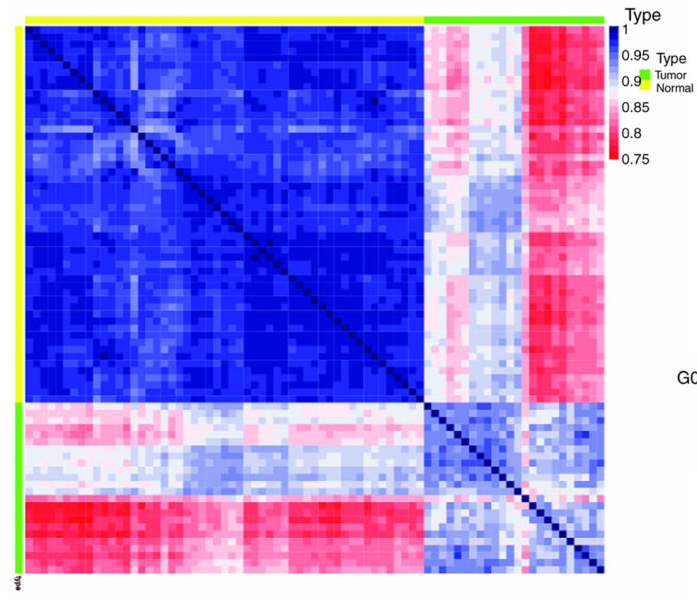

B

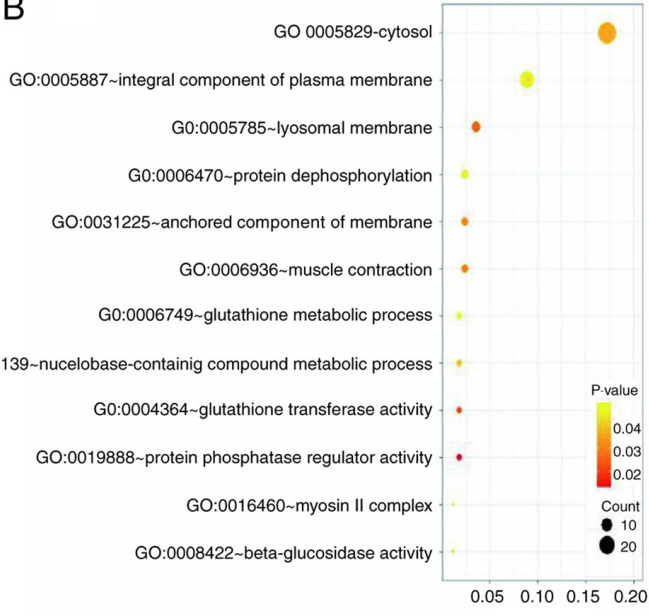

GeneRatio

C

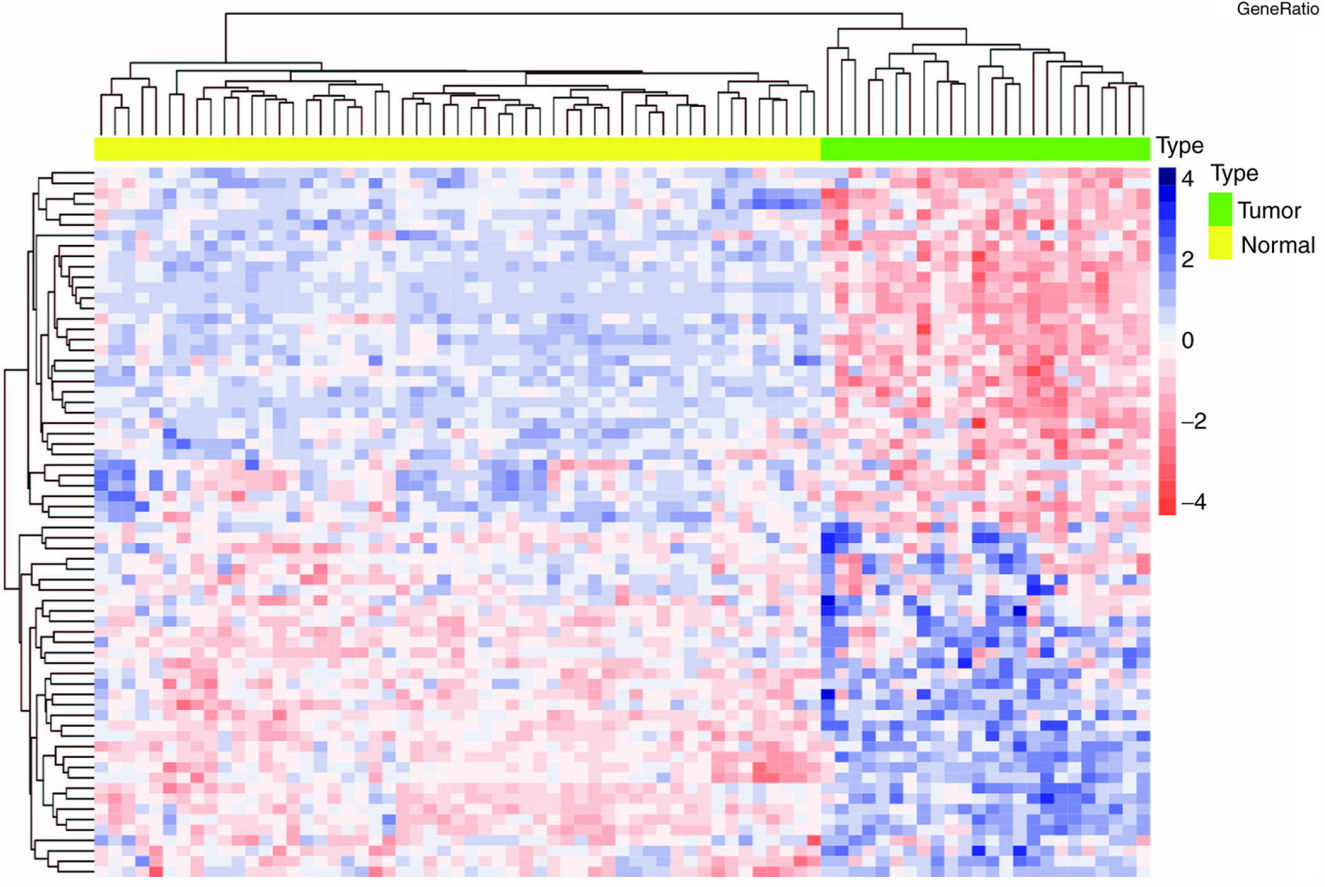

Figure 2. Function analysis of recurrence-associated genes (A) Gene expression heat map of the module most relevant to recurrence in tumor and paracancerous tissues. The yellow label represents the paracancerous samples and the green label represents the tumor tissue samples. The blue/red scale bar represents the expression level of genes. (B) GO enrichment analysis was performed on the 117 genes in the relevant module. The original value outputted from the Database for Annotation, Visualization and Integrated Discovery for GO biological processes was converted into '-log (P-value)' for plotting. (C) Expression heat map of differentially expressed genes in the module most relevant to recurrence in tumor and paracancerous tissues. The yellow label represents the paracancerous samples and the green label represents the tumor tissue samples. The blue/red scale bar represents the expression level of genes. GO, Gene Ontology.

examination was performed every three months from the time of the first repeated examination, which was 90 days after surgery. If the imaging examination indicated that the patient had relapsed, they were classified into the relapsed group (35 patients), while 70 patients did not relapse, and the level of UPK 2 mRNA detected was recorded. If the patient had no recurrence during the 3-year follow-up period, then the mean value of multiple testing was recorded as the relative expression level of UPK2. The results demonstrated no significant differences in UPK2 expression between patients with LUAD of different ages and sex (Fig. 4A and B). Notably, non-relapsed patients exhibited low UPK2 expression. The mean expression level of UPK2 for relapsed patients was measured after recurrence was detected via imaging. The mean UPK2 expression relative to GADPH in relapsed patients was 0.2763 , while the mean UPK2 expression in non-relapsed patients was 0.1623 , which was significantly lower than that of relapsed patients $(\mathrm{P}<0.0001$; Fig. 4C). Notably, for relapsed patients, UPK2 expression at relapse was significantly higher than that before relapse ( $\mathrm{P}=0.0351$; Fig. 4D). In addition, the ROC curve was plotted and the AUC was calculated to determine whether plasma UPK2 expression may be used to distinguish between relapsed and non-relapsed patients (Fig. 4E). The results demonstrated that when plasma UPK2 expression was used alone as a diagnostic biomarker, the AUC was 0.767 with a 95\% CI of 0.675-0.858. Furthermore, patients with LUAD were divided into two groups, namely the high $(\geq 0.1623)$ and low $(<0.1623)$ UPK2 expression groups, and their survival curves were plotted. The results indicated that patients with high plasma UPK2 mRNA expression had a poorer survival 
Table I. UPK2, KLHDC3, GALR2 and TYRP1 expression are highly associated with survival and recurrence.

\begin{tabular}{lccc}
\hline Gene & Nodes no. (power=8) & Probes name used by oncomine database & P-value \\
\hline UPK2 & 15 & 207862 at & $4.9 \times 10^{-06}$ \\
GALR2 & 14 & 211226 at & 0.0093 \\
KLHDC3 & 12 & 208784 _s_at & $1.7 \times 10^{-06}$ \\
TYRP1 & 11 & 205694_at & $2 \times 10^{-07}$ \\
\hline
\end{tabular}

ULPK2, uroplakin 2; KLHDC3, kelch domain containing 3; GALR2, galanin receptor 2; TYRP1, tyrosinase-related protein 1.

A

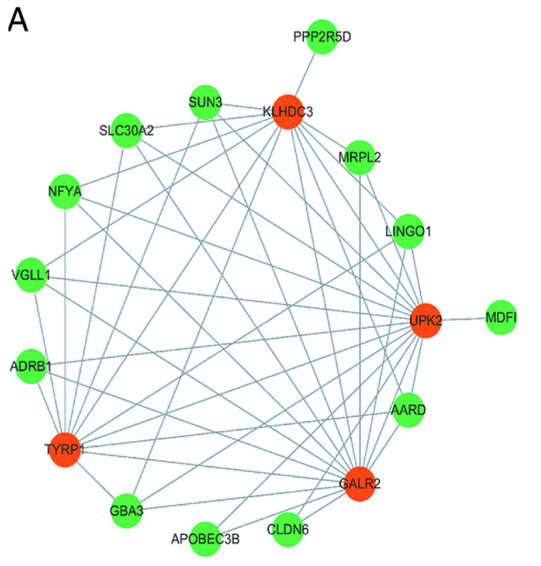

C

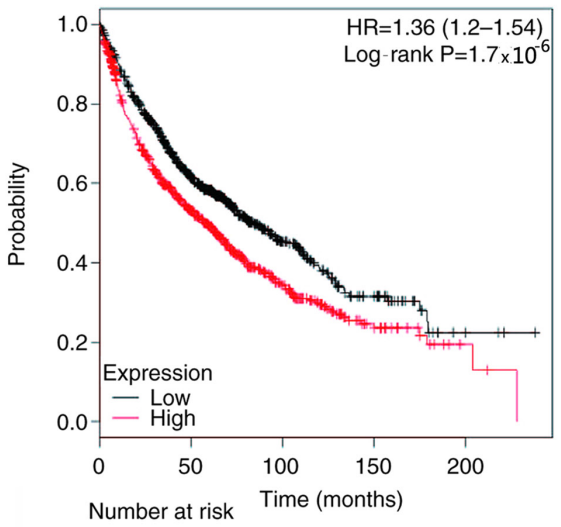

$\begin{array}{lllll}\text { Low } 965 & 462 & 102 & 33 & 4 \\ \text { High } 961 & 366 & 101 & 24 & 3\end{array}$

E

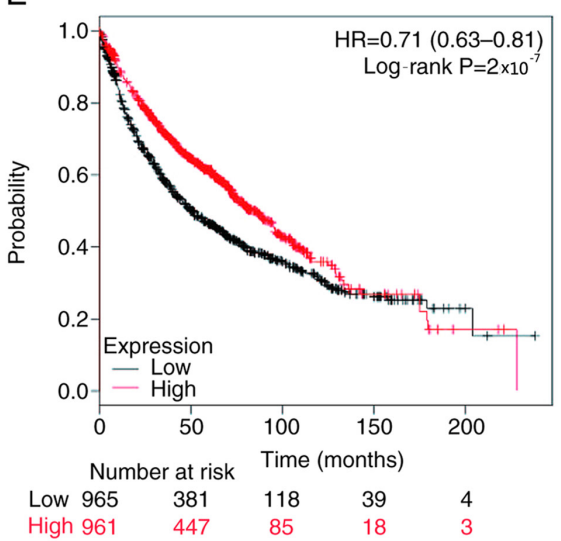

B

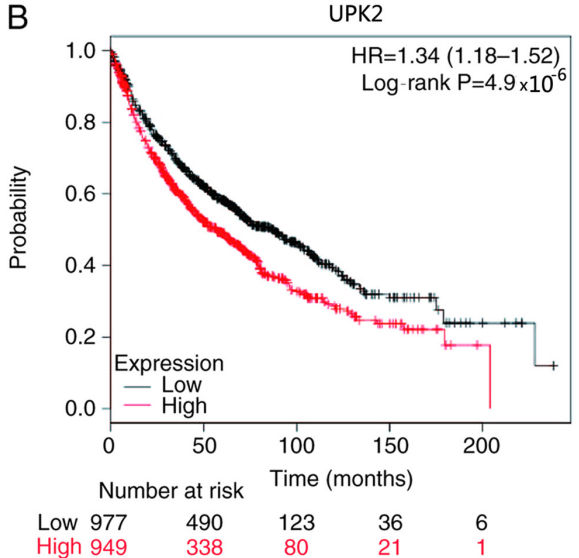

D GALR2

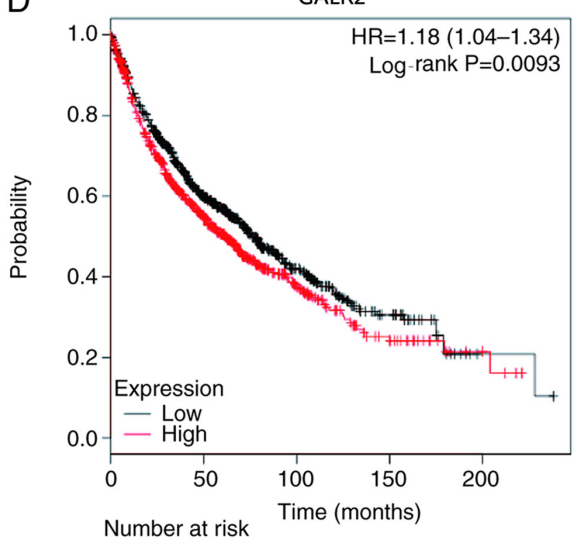

$\begin{array}{lcccc}\text { Low } 978 & 453 & 118 & 33 & 2 \\ \text { High } 948 & 375 & 85 & 24 & 5\end{array}$

Figure 3. Key genes associated with lung adenocarcinoma recurrence in the purple module. (A) Cytoscape that visualizes the co-expression network. The four orange circles represent the four genes with the highest degree of connectivity in the relevant module. Kaplan-Meier survival plots for the overall survival based on the expression levels of (B) UPK2, (C) KLHDC3, (D) GALR2 and (E) TYRP1. ULPK2, uroplakin 2; KLHDC3, kelch domain containing 3; GALR2, galanin receptor 2; TYRP1, tyrosinase-related protein 1 ; HR, hazard ratio. 
Table II. Demographic information and clinical characteristics of 105 patients with lung adenocarcinoma with (n=35) or without $(\mathrm{n}=70)$ recurrence.

\begin{tabular}{|c|c|c|c|}
\hline Characteristics & All & Recurrence & No recurrence \\
\hline \multicolumn{4}{|l|}{ Sex, n $(\%)$} \\
\hline Male & $58(55)$ & $23(66)$ & $35(50)$ \\
\hline Female & $47(45)$ & $12(34)$ & $35(50)$ \\
\hline Median age (range), years & $58(39-83)$ & $59(39-80)$ & $58(42-83)$ \\
\hline \multicolumn{4}{|l|}{ Mortality, n (\%) } \\
\hline Dead & $74(70)$ & $26(74)$ & $48(69)$ \\
\hline Alive & $23(22)$ & $7(20)$ & $16(23)$ \\
\hline Unknown & $8(8)$ & $2(6)$ & $6(9)$ \\
\hline \multicolumn{4}{|l|}{ Smoking status, n (\%) } \\
\hline Former & $70(67)$ & $22(63)$ & $48(69)$ \\
\hline Active & $25(24)$ & $11(31)$ & $14(20)$ \\
\hline Never & $8(8)$ & $2(6)$ & $6(9)$ \\
\hline Unknown & $2(2)$ & $0(0)$ & $2(3)$ \\
\hline \multicolumn{4}{|l|}{ Stage at diagnosis, n (\%) } \\
\hline I & $65(62)$ & $21(60)$ & $44(63)$ \\
\hline II & $22(21)$ & $10(29)$ & $12(17)$ \\
\hline IIIA & $18(17)$ & $4(11)$ & $14(20)$ \\
\hline \multicolumn{4}{|l|}{ Treatment, n (\%) } \\
\hline No adjuvant treatment & $62(59)$ & $16(46)$ & $46(66)$ \\
\hline Adjuvant treatment & $43(41)$ & $19(54)$ & $24(34)$ \\
\hline Adjuvant chemotherapy & $4(4)$ & $2(6)$ & $2(3)$ \\
\hline Radiation & $35(33)$ & $15(43)$ & $20(27)$ \\
\hline Radiation and chemotherapy & $4(4)$ & $2(6)$ & $2(3)$ \\
\hline Mean uroplakin 2 expression (range) & $0.20(0.05-0.80)$ & $0.28(0.08-0.61)$ & $0.16(0.05-0.80)$ \\
\hline
\end{tabular}

outcome than those with low plasma UPK2 mRNA expression (Fig. 4F).

\section{Discussion}

Lung cancer has become a global health problem due to the high morbidity and mortality rates $(0.057$ and $85 \%$, respectively) in China in 2017 (2,3). LUAD is the main subtype of NSCLC (31-33). Despite recent advancements in cancer treatment, the 5-year survival rate of patients with LUAD remains relatively low $(\sim 15 \%)(34,35)$. With the advent of precision medicine concepts, molecular biomarkers and molecular drug targets have become hotspots in cancer research, improving the long-term outcomes for patients with different types of cancer. For example, patients with lung cancer with EGFR mutations can benefit from the treatment of tyrosine kinase inhibitors, such as gefitinib and erlotinib (36). Other potential biomarkers are predominantly oncogene-driven mutations, including ALK translocation and ROS1 gene rearrangement $(37,38)$. Thus, it remains critical to identify and validate clinically relevant and effective prognostic markers for LUAD to complement existing molecular biomarkers and further guide treatment decisions.

UPK 2 is a highly specific marker of bladder transitional cell carcinoma. UPK2 mRNA expression was initially detected in blood samples from two patients with metastatic bladder cancer who did not receive chemotherapy and 1 out of 8 patients with metastatic bladder cancer who received chemotherapy (39). However, it was not detected in patients with non-metastatic bladder cancer or in the normal control group, indicating that detection of UPK 2 in the peripheral blood is associated with metastasis of bladder cancer (39). Therefore, assessment of UPK2 specificity and sensitivity may be a potential means of detecting bladder cancer metastasis, staging and monitoring chemotherapy response. Lotan et al (40) assessed 11 immunohistochemical markers at the primary sites of several micropapillary carcinomas and demonstrated that urinary tract proteins can be used as markers to identify urinary mesothelial invasive micropapillary carcinoma. Li et al (41) reported that UPK2 is expressed in $63 \%$ of plasma cell samples, which is significantly higher than UPK3 expression (6\%), and further suggested that UPK2 is a valuable marker and should be included in immunohistochemical markers to facilitate the differential diagnosis of tumors with plasmacytoid features. Furthermore, Matuszewski et al (42) demonstrated that the concentration of UPK2 in urine decreased with the progression of bladder cancer, which further confirms the diagnostic value of UPK2 concentration in plasma and urine for bladder cancer. Tian et al (43) assessed UPK2 expression via bladder tissue microarray and reported that UPK2 is highly specific (100\%) and can be used as a marker to identify urothelial lineage tumors and to help distinguish between bladder and prostate cancers, or 

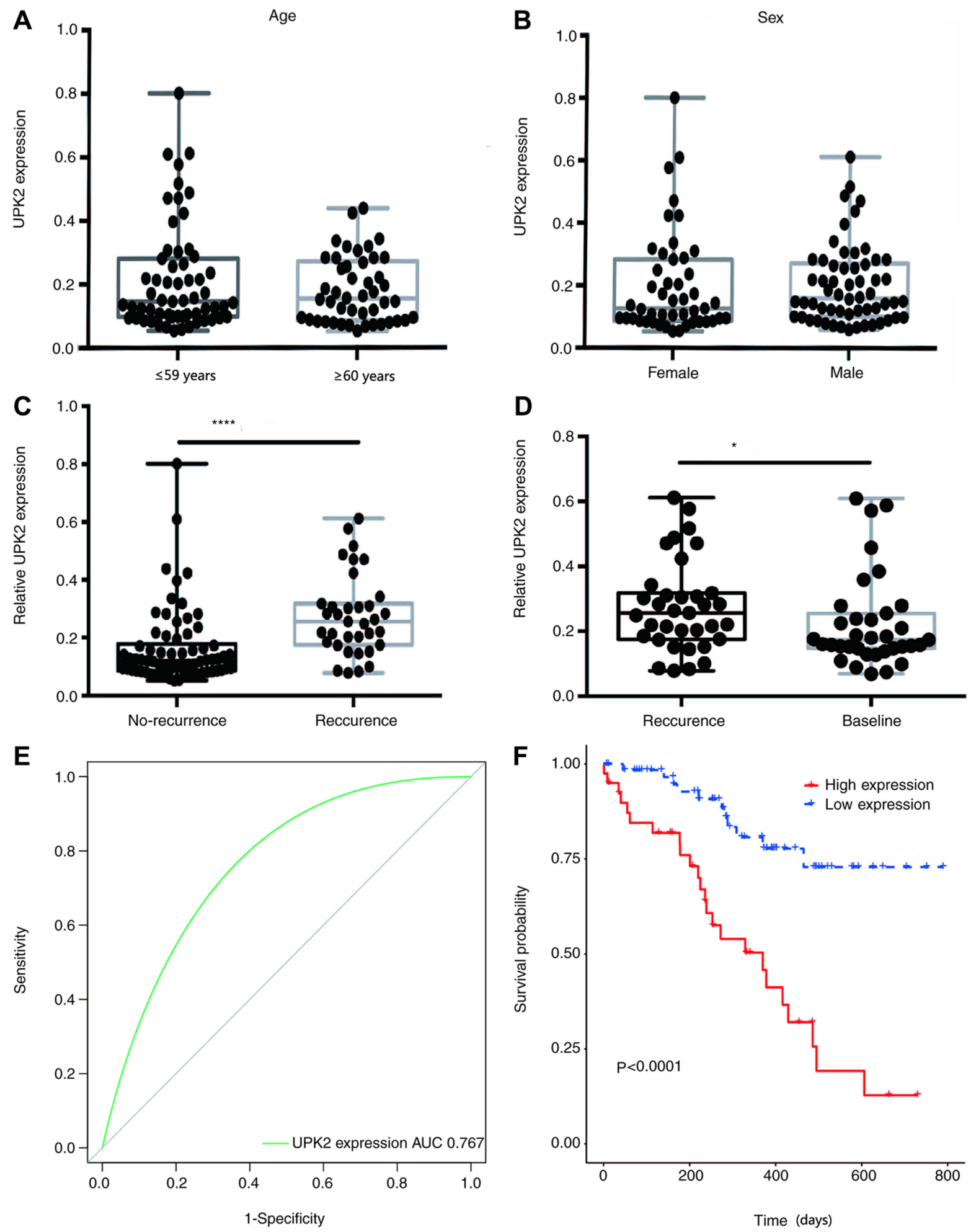

Figure 4. Diagnostic performance of UPK2 in patients with LUAD. Scatter plots of UPK 2 expression in patients with LUAD of (A) different ages and (B) different sex. (C) Scatter plot of UPK2 expression in the relapsed and non-relapsed patients with LUAD. ${ }^{* * * *} \mathrm{P}<0.0001$. (D) Scatter plot of UPK2 expression in patients with LUAD before (baseline) and after relapse. ${ }^{*} \mathrm{P}<0.05$. (E) Diagnostic performance of plasma cell-free UPK2 mRNA for recurrence detection in LUAD. (F) Kaplan-Meier survival plot for overall survival in patients with LUAD with different UPK2 expression. LUAD, lung adenocarcinoma; AUC, area under the curve; UPK2, uroplakin 2.

can be used in combination with GATA3 as a potential marker for metastatic breast cancer. Hoang et al (44) demonstrated that the positive rate of UPK2, GATA3 and p40 antibody combined testing was $94.2 \%(97 / 103)$ in invasive urothelial carcinoma, indicating that combination of these three antibodies has a high sensitivity to the differential diagnosis of invasive urothelial carcinoma. However, the combination testing of UPK2, GATA3 and p40 is negative in LUAD, colon adenocarcinoma and renal cell carcinoma (44). Furthermore, the expression and role of UPK2 in LUAD recurrence has not been fully investigated. 
The results of the present study demonstrated that UPK2 expression was significantly increased in patients with LUAD recurrence, and the prognosis of patients with high UPK2 expression was poor. These different expression levels of UPK2 before and after LUAD recurrence are consistent with the aforementioned findings on bladder cancer, suggesting that the differential expression of UPK 2 in patients with LUAD recurrence may have clinical implications (39). Enrichment analysis demonstrated that the function of UPK2 was predominantly associated with 'cytosol'. Blood samples were collected from 105 patients with LUAD, of which 35 had LUAD recurrence and 70 patients had no recurrence. RT-qPCR analysis demonstrated that UPK2 mRNA expression in the blood of relapsed patients was significantly higher than that of patients without recurrence, indicating that the difference in UPK2 expression was significantly associated with the recurrence of LUAD. Therefore, the present results suggested that UPK 2 may be used as a biomarker to detect recurrence instead of using imaging techniques, since the detection of free UPK2 mRNA is easier to perform and less invasive. Prospective studies should further investigate UPK2 expression in patients with LUAD, with different stages and lymph node metastasis, and should further validate the clinicopathological characteristics associated with UPK2 expression, in order to develop novel therapeutic strategies for patients with LUAD.

\section{Acknowledgements}

Not applicable.

\section{Funding}

This study was funded by National Natural Science Foundation of China (grant no. 81672890).

\section{Availability of data and materials}

The datasets used and/or analyzed during the current study are available from the corresponding author on reasonable request.

\section{Authors' contributions}

$\mathrm{JZ}$ performed the experiments, prepared the figures and wrote the manuscript. QL and BL performed clinical sample collection and preparation. HL and CW performed the bioinformatics analysis. CL and HJ interpreted the data, drafted and revised the manuscript, and gave final approval of the version to be published. All authors read and approved the final manuscript.

\section{Ethics approval and consent to participate}

The study was approved by the Ethics Committee and Institutional Review Board of the First Affiliated Hospital of the Second Military Medical University (approval no. CHEC2020-021). All patients provided oral informed consent.

\section{Patient consent for publication}

Not applicable.

\section{Competing interests}

The authors declare that they have no competing interests.

\section{References}

1. Zhu Y, Xing P, Wang S, Ma D, Mu Y, Li X, Xu Z and Li J: Evaluation of calculating carboplatin dosage in carboplatin-pemetrexed therapy as the first-line therapy for Chinese patients with advanced lung adenocarcinoma. Thorac Cancer 9: 400-407, 2018

2. Liu AN, Qu HJ, Yu CY and Sun P: Knockdown of LINC01614 inhibits lung adenocarcinoma cell progression by up-regulating miR-217 and down-regulating FOXP1. J Cell Mol Med 22: 4034-4044, 2018.

3. Zhou X, Zhang P, Luo W, Zhang L, Hu R, Sun Y and Jiang H: Ketamine induces apoptosis in lung adenocarcinoma cells by regulating the expression of CD69. Cancer Med 7: 788-795, 2018.

4. Han B, Jin B, Chu T, Niu Y, Dong Y, Xu J, Gu A, Zhong H, Wang $\mathrm{H}$, Zhang X, et al: Combination of chemotherapy and gefitinib as first-line treatment for patients with advanced lung adenocarcinoma and sensitive EGFR mutations: A randomized controlled trial. Int J Cancer 141: 1249-1256, 2017.

5. Zhuang X, Qiao T, Xu G, Yuan S, Zhang Q and Chen X: Combination of nadroparin with radiotherapy results in powerful synergistic antitumor effects in lung adenocarcinoma A549 cells. Oncol Rep 36: 2200-2206, 2016.

6. Noronha V, Zanwar S, Joshi A, Patil VM, Mahajan A, Janu A, Agarwal JP, Bhargava P, Kapoor A and Prabhash K: Practice patterns and outcomes for pemetrexed plus platinum doublet as neoadjuvant chemotherapy in adenocarcinomas of lung: Looking beyond the usual paradigm. Clin Oncol (R Coll Radiol) 30: 23-29, 2018.

7. Marini KD, Croucher DR, McCloy RA, Vaghjiani V, Gonzalez-Rajal A, Hastings JF, Chin V, Szczepny A, Kostyrko K, Marquez C, et al: Inhibition of activin signaling in lung adenocarcinoma increases the therapeutic index of platinum chemotherapy. Sci Transl Med 10: eaat3504, 2018.

8. Tao H, Meng Q, Li M, Shi L, Tang J and Liu Z: Outcomes of bevacizumab combined with chemotherapy in lung adenocarcinoma-induced malignant pleural effusion. Thorac Cancer 9: 298-304, 2018.

9. Wu K, Zhang X, Li F, Xiao D, Hou Y, Zhu S, Liu D, Ye X, Ye M, Yang $\mathrm{J}$, et al: Frequent alterations in cytoskeleton remodelling genes in primary and metastatic lung adenocarcinomas. Nat Commun 6: 10131, 2015.

10. Wang X, Xu W, Zhan P, Xu T, Jin J, Miu Y, Zhou Z, Zhu Q, Wan B, Xi G, et al: Overexpression of geranylgeranyl diphosphate synthase contributes to tumour metastasis and correlates with poor prognosis of lung adenocarcinoma. J Cell Mol Med 22: 2177-2189, 2018

11. Zhao S, Guo W, Li J, Yu W, Guo T, Deng W and Gu C: High expression of Y-box-binding protein 1 correlates with poor prognosis and early recurrence in patients with small invasive lung adenocarcinoma. Onco Targets Ther 9: 2683-2692, 2016.

12. Mansoori B, Mohammadi A, Davudian S, Shirjang S and Baradaran B: The different mechanisms of cancer drug resistance: A brief review. Adv Pharm Bull 7: 339-348, 2017.

13. Zhang C, Leighl NB, Wu YL and Zhong WZ: Emerging therapies for non-small cell lung cancer. J Hematol Oncol 12: 45, 2019.

14. Aramini B, Casali C, Stefani A, Bettelli S, Wagner S, Sangale Z, Hughes E, Lanchbury JS, Maiorana A and Morandi U: Prediction of distant recurrence in resected stage I and II lung adenocarcinoma. Lung Cancer 101: 82-87, 2016.

15. Krishnamurthy J, Torrice C, Ramsey MR, Kovalev GI, Al-Regaiey K, Su L and Sharpless NE: Ink4a/Arf expression is a biomarker of aging. J Clin Invest 114: 1299-1307, 2004.

16. Shaw LM, Vanderstichele H, Knapik-Czajka M, Clark CM, Aisen PS, Petersen RC, Blennow K, Soares H, Simon A, Lewczuk $\mathrm{P}$, et al: Cerebrospinal fluid biomarker signature in Alzheimer's disease neuroimaging initiative subjects. Ann Neurol 65: 403-413, 2009.

17. Ji X, Takahashi R, Hiura Y, Hirokawa G, Fukushima Y and Iwai N: Plasma miR-208 as a biomarker of myocardial injury. Clin Chem 55: 1944-1949, 2009.

18. Plotti F, Capriglione S, Terranova C, Montera R, Aloisi A, Damiani P, Muzii L, Scaletta G, Benedetti-Panici P and Angioli R: Does HE4 have a role as biomarker in the recurrence of ovarian cancer? Tumour Biol 33: 2117-2123, 2012. 
19. Huang Y, Cen J, Wei J, Chen Z, Fang Y, Feng Z, Lu J, Liang Y, Luo J, Mo C and Chen W: Impact of AIB1 expression on the prognosis of upper tract urothelial carcinoma after radical nephroureterectomy. Cancer Biomark 25: 151-160, 2019.

20. Sgroi DC, Carney E, Zarrella E, Steffel L, Binns SN, Finkelstein DM, Szymonifka J, Bhan AK, Shepherd LE, Zhang Y, et al: Prediction of late disease recurrence and extended adjuvant letrozole benefit by the HOXB13/IL17BR biomarker. J Natl Cancer Inst 105: 1036-1042, 2013.

21. Ni KW and Sun GZ: The identification of key biomarkers in patients with lung adenocarcinoma based on bioinformatics. Math Biosci Eng 16: 7671-7687, 2019.

22. LiR, Yang YE, Yin YH,Zhang MY, Li H and Qu YQ: Methylation and transcriptome analysis reveal lung adenocarcinoma-specific diagnostic biomarkers. J Transl Med 17: 324, 2019.

23. Langfelder P and Horvath S: WGCNA: An R package for weighted correlation network analysis. BMC Bioinformatics 9: 559, 2008.

24. Langfelder P and Horvath S: Fast R functions for robust correlations and hierarchical clustering. J Stat Softw 46: i11, 2012.

25. Livak KJ and Schmittgen TD: Analysis of relative gene expression data using real-time quantitative PCR and the 2(-Delta Delta C(T)) method. Methods 25: 402-408, 2001.

26. Robin X, Turck N, Hainard A, Tiberti N, Lisacek F, Sanchez JC and Müller M: pROC: An open-source package for R and S+ to analyze and compare ROC curves. BMC Bioinformatics 12: 77, 2011.

27. Yu L, Tao G, Zhu L, Wang G, Li Z, Ye J and Chen Q: Prediction of pathologic stage in non-small cell lung cancer using machine learning algorithm based on CT image feature analysis. BMC Cancer 19: 464, 2019.

28. Mähler N, Wang J, Terebieniec BK, Ingvarsson PK, Street NR and Hvidsten TR: Gene co-expression network connectivity is an important determinant of selective constraint. PLoS Genet 13 e1006402, 2017

29. Miura N, Hasegawa J and Shiota G: Serum messenger RNA as a biomarker and its clinical usefulness in malignancies. Clin Med Oncol 2: 511-527, 2008

30. Tani N, Ichikawa D, Ikoma D, Tomita H, Sai S, Ikoma H, Okamoto K, Ochiai T, Ueda Y, Otsuji E, et al: Circulating cell-free mRNA in plasma as a tumor marker for patients with primary and recurrent gastric cancer. Anticancer Res 27: $1207-1212,2007$

31. Siegel RL, Miller KD and Jemal A: Cancer statistics, 2017. CA Cancer J Clin 67: 7-30, 2017.

32. Youlden DR, Cramb SM and Baade PD: The international epidemiology of lung cancer: Geographical distribution and secular trends. J Thorac Oncol 3: 819-831, 2008.

33. Rosell R, Bivona TG and Karachaliou N: Genetics and biomarkers in personalisation of lung cancer treatment. Lancet 382: 720-731, 2013.

34. Hung JJ, Yeh YC, Jeng WJ, Wu KJ, Huang BS, Wu YC, Chou TY and Hsu WH: Predictive value of the international association for the study of lung cancer/American Thoracic Society/European Respiratory Society classification of lung adenocarcinoma in tumor recurrence and patient survival. J Clin Oncol 32: $2357-2364,2014$.
35. Subramaniam S, Thakur RK, Yadav VK, Nanda R, Chowdhury S and Agrawal A: Lung cancer biomarkers: State of the art. J Carcinog 12: 3, 2013

36. Remon J, Moran T, Reguart N, Majem M, Carcereny E and Lianes P: Beyond EGFR TKI in EGFR-mutant non-small cell lung cancer patients: Main challenges still to be overcome. Cancer Treat Rev 40: 723-729, 2014

37. Soda M, Choi YL, Enomoto M, Takada S, Yamashita Y, Ishikawa S, Fujiwara S, Watanabe H, Kurashina K, Hatanaka H, et al: Identification of the transforming EML4-ALK fusion gene in non-small-cell lung cancer. Nature 448: 561-566, 2007.

38. Bergethon K, Shaw AT, Ou SH, Katayama R, Lovly CM, McDonald NT, Massion PP, Siwak-Tapp C, Gonzalez A, Fang R, et al: ROS1 rearrangements define a unique molecular class of lung cancers. J Clin Oncol 30: 863-870, 2012.

39. Li SM, Zhang ZT, Chan S, McLenan O, Dixon C, Taneja S, Lepor H, Sun TT and Wu XR: Detection of circulating uroplakin-positive cells in patients with transitional cell carcinoma of the bladder. J Urol 162(3 Pt 1): 931-935, 1999.

40. Lotan TL, Ye H, Melamed J, Wu XR, Shih IM and Epstein JI: Immunohistochemical panel to identify the primary site of invasive micropapillary carcinoma. Am J Surg Pathol 33: 1037-1041, 2009.

41. Li W, Liang Y, Deavers MT, Kamat AM, Matin SF, Dinney CP, Czerniak B and Guo CC: Uroplakin II is a more sensitive immunohistochemical marker than uroplakin III in urothelial carcinoma and its variants. Am J Clin Pathol 142: 864-871, 2014

42. Matuszewski M, Szymanska B, Dlugosz A, Malkiewicz B, Dembowski J and Piwowar A: Preliminary evaluation of the diagnostic usefulness of uroplakin 2 with an assessment of the antioxidant potential of patients with bladder cancer. Biomed Res Int 2018: 8693297, 2018

43. Tian W, Guner G, Miyamoto H, Cimino-Mathews A, Gonzalez-Roibon N, Argani P, Li X, Sharma R, Subhawong AP, Rezaei K, et al: Utility of uroplakin II expression as a marker of urothelial carcinoma. Hum Pathol 46: 58-64, 2015.

44. Hoang LL, Tacha D, Bremer RE, Haas TS and Cheng L: Uroplakin II (UPII), GATA3, and p40 are highly sensitive markers for the differential diagnosis of invasive urothelial carcinoma. Appl Immunohistochem Mol Morphol 23: 711-716, 2015.

This work is licensed under a Creative Commons Attribution-NonCommercial-NoDerivatives 4.0 International (CC BY-NC-ND 4.0) License. 